Section Editor Mitchell S.V. Elkind, MD, MS

\title{
Emerging Subspecialties in Neurology: Transcranial stimulation
}

Luca Peruzzotti-Jametti, MD

Marco Bacigaluppi, MD

Stefano Sandrone

Marco Cambiaghi, PhD

Correspondence to

Dr. Cambiaghi:

marco.cambiaghi@unito.it
Treating neurologic disorders with noninvasive brain stimulation techniques has always been one of the major goals of neurophysiology and to a broad extent, of modern neurology and psychiatry. The first attempts date back at least to $50 \mathrm{AD}$, when the Roman physician Scribonius Largus used electric currents from torpedo fishes to treat headaches. ${ }^{1}$ Unfortunately, these initial efforts sank into oblivion until the 18th century. In the last 2 decades, thanks to technological improvements, noninvasive transcranial stimulation is re-emerging as an appealing and promising approach to be used in different fields of neurology and neuroscience, with the number of related publications increasing exponentially in both clinical and basic science studies. This new age is particularly due to the broad-spectrum use of 2 techniques called transcranial magnetic stimulation (TMS) and transcranial direct current stimulation (tDCS). Despite the undoubted importance of conventional brain stimulation methods such as deep brain stimulation (DBS) or electroconvulsive therapy, TMS and tDCS present many favorable advantages both in terms of noninvasiveness and safety, with minimal or no side effects.

TMS is based on the induction of single or repetitive (rTMS) pulses of electric current within the brain, obtained by generating magnetic fields near the scalp through the application of an electromagnetic coil. Differently, tDCS involves the application of a weak, transcranially delivered direct current, flowing from positive (anodal) to negative (cathodal) poles. TMS allows a good spatial and temporal resolution whereas tDCS advantages rely on portability, reduced costs, and ease of combination with other treatments (e.g., rehabilitation sessions). Both rTMS and tDCS have neuromodulatory effects on cortical excitability that can outlast the stimulation period, depending on timing, intensity, and frequency/polarity. While lowfrequency rTMS $(<2 \mathrm{~Hz})$ and cathodal tDCS reduce cortical excitability, high-frequency rTMS $(>5 \mathrm{~Hz})$ and anodal tDCS have excitatory effects.

STIMULATING CHALLENGES Transcranial stimulation poses several opportunities for neurologist training to gain insights in different experimental and clinical applications for the treatment of various neurologic disorders.

In mood disorders, there is evidence of an unbalanced cortical activity in the prefrontal cortex, with a relatively decreased activity on the left side which can be rebalanced with focal stimulation of the dorsolateral prefrontal cortex. ${ }^{2}$ Symptomatic improvements of untreatable depression were so substantial that, in 2008, the Food and Drug Administration approved rTMS for nonresponders to conventional pharmacologic therapies.

In addition to the established effectiveness of DBS techniques in selected patients with Parkinson disease, tDCS is able to reduce bradykinesia and ameliorate gait. ${ }^{3}$ Even though larger studies are needed to confirm these effects of transcranial stimulation, its noninvasiveness is paving the way for a new therapeutic option, which will be available for a large number of patients.

The application of these neuromodulatory techniques to target chronic pain syndromes appears very promising. Transcranial stimulation is able to prevent maladaptive plastic changes underpinning chronic pain, and the interactions between cerebral stimulation and analgesics will be one of the next frontiers of investigation. ${ }^{4}$

Exploiting the focality of rTMS is useful in focal and to a lesser extent in generalized epilepsy. However, the poor correlation between real seizure frequency and those reported by patients makes the boundary of a possible placebo effect difficult to find. ${ }^{5}$

There is also increasing evidence that transcranial stimulation might improve selective skills in cognitive disorders such as Alzheimer disease. These findings are very preliminary, since improving performances in a single task does not necessarily represent a global cognitive enhancement. ${ }^{6}$

With respect to these chronic neurologic disorders, the application of rTMS and tDCS in monophasic diseases such as ischemic stroke holds great promise. The ability of transcranial stimulation to enhance (or reduce) the cortical activity of the lesioned (or the unaffected) hemisphere has been used to ameliorate motor impairment, poststroke depression, and cortical deficits

From Vita-Salute San Raffaele University (L.P.-J., M.B., S.S.), Neuroimmunology Unit (L.P.-J., M.B., S.S.), and Experimental Neurophysiology Unit (M.C.), Institute of Experimental Neurology (INSPE), Division of Neuroscience, DIBIT-II, San Raffaele Scientific Institute, Milan, Italy. Dr. Cambiaghi is currently with the Department of Neuroscience, University of Turin.

Go to Neurology.org for full disclosures. Funding information and disclosures deemed relevant by the authors, if any, are provided at the end of the article. 
in chronic stroke patients. ${ }^{7}$ Currently, one approach triggers long-lasting modifications of neuronal circuits in the ischemic hemisphere; an alternative approach is to modulate re-adaptive beneficial processes that seem to be hampered by an inhibition from the contralesional unaffected hemisphere. Nonetheless, different approaches and earlier times of application may represent future fields of investigation.

Far from being a panacea, transcranial stimulation has shown several advantages that will expand more and more treatment options for many cases of pharmacologically untreatable diseases, including mood and movement disorders, chronic pain, epilepsy, dementia, stroke, and other neurologic disorders.

AN INTERDISCIPLINARY FIELD Residents who wish to be effective in transcranial stimulation need to weave strict interconnections with specialists in neurorehabilitation, psychology, and neuroimaging to disclose underlying deficiencies and selectively target treatments.

Recently, one of the most exciting areas of investigation has been uncovering the in vivo functional and structural effects of transcranial stimulation. Neuroimaging is currently used to indirectly explore synaptic activity through the measurement of cerebral blood flow and metabolism. These techniques are giving precious insights into the biological effects of transcranial stimulation and will help to define the correct time window for treatments. In parallel, structural MRI techniques, which are able to assess the integrity of white matter bundles, will probably be fundamental in the polimodal pretherapy assessments of patients to be stimulated. ${ }^{8}$ In the next few years, advanced MRI techniques will become a pivotal tool for the development of individualized therapeutic regimens and also provide new biomarkers of clinical outcome and follow-up.

Future investigations will also have to elucidate the physiologic and molecular mechanisms of action of transcranial stimulation. Actually, it has been demonstrated that in rodents both rTMS and tDCS have effects similar to those observed in humans ${ }^{9}$; however, detailed mechanistic insights are still missing and will probably derive from further animal studies. Working with transcranial stimulation will require a strong collaboration among clinicians of different disciplines and translational research programs, so to facilitate bench-to-bedside translation in developing cohesive patient-oriented treatment plans.

TRAINING AND CAREER OPPORTUNITIES With the advancements in understanding micro and macro plasticity of the brain tissue, as well as technical advancements, transcranial stimulation offers a unique opportunity for clinicians interested in participating in the development of novel treatment protocols. Notably, at the time of writing, 495 clinical trials including transcranial stimulation treatment have been registered on the NIH (see the www.clinicaltrials.gov Web site). Of these trials, about 30 concern chronic pain, 33 cognitive disorders, 38 movement disorders, 85 disability related to ischemic stroke, and 128 psychiatric disorders. Remarkably, out of the 203 completed trials, only a minority have already been published. Since the other 213 trials are still recruiting patients, the near future will reveal to us the efficacy and safety of many transcranial treatment protocols. In fact, clinical updates and experimental progresses are continuously reported in various scientific journals dedicated to neurophysiology. Remarkably, entire books devoted to transcranial stimulation as well as a recently launched peer-reviewed journal restricted to the field (Brain Stimulation) are available. ${ }^{10}$ In addition, a dedicated conference on Transcranial Magnetic and Direct Current Stimulation was established since 1998, and more and more network groups are emerging. For residents, the best way to approach these techniques is to initially enroll in a neurophysiological department encompassing a well-established transcranial stimulation laboratory. Fellows will have to learn the basic diagnostic tools of neurophysiology and their acquired knowledge will be fundamental to develop new insights in transcranial stimulation as well as new therapeutic strategies. Interested residents will have to take charge of establishing their own training path-daunting but well worth it.

DISCUSSION Transcranial stimulation is an emerging field of clinical neurophysiology, aimed at ameliorating disability of various neurologic diseases. Technological improvements, the disclosures of ongoing trials, and the need to set consensus-based clinical protocols are good premises to jolt the boundaries of transcranial stimulation. It is therefore reasonable to think that the best-for patients, young neurologists, and physician scientists-is yet to come.

\section{AUTHOR CONTRIBUTIONS}

Luca Peruzzotti-Jametti, Marco Bacigaluppi, Stefano Sandrone, and Marco Cambiaghi wrote the article.

\section{ACKNOWLEDGMENT}

The authors thank Jenna Nankin for editing assistance and Dr. Letizia Leocani, Dr. Gianvito Martino, and Prof. Giancarlo Comi for mentoring.

\section{STUDY FUNDING}

No targeted funding reported.

\section{DISCLOSURE}

The authors report no disclosures relevant to the manuscript. Go to Neurology.org for full disclosures.

\section{REFERENCES}

1. Priori A. Brain polarization in humans: a reappraisal of an old tool for prolonged non-invasive modulation of brain excitability. Clin Neurophysiol 2003;114:589-595. 
2. Hamilton R, Messing S, Chatterjee A. Rethinking the thinking cap: ethics of neural enhancement using noninvasive brain stimulation. Neurology 2011;76:187-193.

3. Benninger DH, Lomarev M, Lopez G, et al. Transcranial direct current stimulation for the treatment of Parkinson's disease. J Neurol Neurosurg Psychiatry 2010;81:1105-1111.

4. Fregni F, Pascual-Leone A. Technology insight: noninvasive brain stimulation in neurology-perspectives on the therapeutic potential of rTMS and tDCS. Nat Clin Pract Neurol 2007;3:383-393.

5. Nitsche MA, Paulus W. Noninvasive brain stimulation protocols in the treatment of epilepsy: current state and perspectives. Neurotherapeutics 2009;6:244-250.

6. Nardone R, Bergmann J, Christova M, et al. Effect of transcranial brain stimulation for the treatment of
Alzheimer disease: a review. Int J Alzheimers Dis 2012;2012:687909.

7. Schlaug G, Renga V, Nair D. Transcranial direct current stimulation in stroke recovery. Arch Neurol 2008;65:1571-1576.

8. Venkatakrishnan A, Sandrini M. Combining transcranial direct current stimulation and neuroimaging: novel insights in understanding neuroplasticity. J Neurophysiol 2012;107:1-4.

9. Cambiaghi M, Teneud L, Velikova S, et al. Flash visual evoked potentials in mice can be modulated by transcranial direct current stimulation. Neuroscience 2011;185:161-165.

10. Brunoni AR, Nitsche MA, Bolognini N, et al. Clinical research with transcranial direct current stimulation (tDCS): challenges and future directions. Brain Stimul 2012;5:175-195. 


\title{
Neurology
}

\author{
Emerging subspecialties in Neurology: Transcranial stimulation \\ Luca Peruzzotti-Jametti, Marco Bacigaluppi, Stefano Sandrone, et al. \\ Neurology 2013;80;e33-e35 \\ DOI 10.1212/WNL.0b013e3182833d74
}

This information is current as of January 21, 2013

\section{Updated Information \&} Services

References

Permissions \& Licensing

Reprints including high resolution figures, can be found at: http://n.neurology.org/content/80/4/e33.full

This article cites 10 articles, 2 of which you can access for free at: http://n.neurology.org/content/80/4/e33.full\#ref-list-1

Information about reproducing this article in parts (figures,tables) or in its entirety can be found online at:

http://www.neurology.org/about/about_the_journal\#permissions

Information about ordering reprints can be found online:

http://n.neurology.org/subscribers/advertise

Neurology ${ }^{\circledR}$ is the official journal of the American Academy of Neurology. Published continuously since 1951 , it is now a weekly with 48 issues per year. Copyright @ 2013 American Academy of Neurology. All rights reserved. Print ISSN: 0028-3878. Online ISSN: 1526-632X.

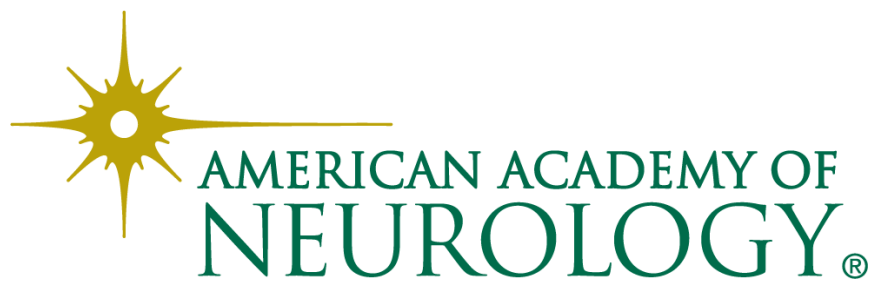

